\title{
Deactivation in the Rabbit Left Ventricle Induced by Constant Ejection Flow
}

\author{
HESSEL WIJKSTRA AND HERMAN B. K. BOOM
}

\begin{abstract}
Pressure generated by the left ventricle after ejection with constant flow was studied for different values of the ejection flow, flow duration, time of flow arrest, and ventricular volume. It was found that pressure after ejection, normalized with respect to isovolumic pressure at the same volume, is regenerated according to a model consisting of an elastance, a resistance, a series elastance, and an additional deactivation component. Deactivation is defined as the difference between the value 1 and the plateau value of the normalized pressure after constant flow ejection. It is shown that this plateau value is constant after constant flow ejection until the minimum in isovolumic $d P / d t$, i.e., during physiological systole. The plateau value is uniquely related to the value of the normalized pressure at the end of a constant flow ejection. After the end of flow, the plateau is reached after an exponential increase of normalized pressure with a time constant of $10.44 \pm 0.09 \mathrm{~ms}$ which agrees with the series-elastance time constant of $10.35 \pm 0.26 \mathrm{~ms}$. These results are discussed with respect to previously found results, and to the implication for predicting pressure generation during physiological flow patterns.
\end{abstract}

\section{INTRODUCTION}

$I^{\prime}$ T has recently been shown that the left ventricle, during and before ejecting with constant flow, exhibits a unique relation between pressure, volume, and rate of volume change (flow) [32], [38]. The pressure-volume component of this relationship has been described in terms of ventricular elastance $E[34]$. The pressure-flow component likewise can be interpreted as a ventricular resistance [16], [32], [38]. In addition, a second much stiffer elastic component $\left(E_{s}\right)$ was found [30], [38], with the help of rapid changes in volume or short flow pulses.

The capability of the ventricle of generating pressure is equivalent to the fact that elastance $E$ is time dependent. Thus, isovolumic generated pressure rises or declines when $E$ rises or declines by $P(t)=E(t) \cdot V(t)$. Therefore, $E(t)$ also represents the time course of isovolumic pressure. Series-elastance likewise changes in time, thus suggesting that it resides in the contractile mechanism of the myocardium. Thus, during isovolumic contraction, the "elastance compartment" of the ventricle does not change

Manuscript received May 13, 1988; revised February 13, 1989. This work was supported in part by The Netherlands Organization for Scientific Research (NWO). Parts of this paper were presented at the 7 th International Conference and Workshop of the Cardiovascular System Dynamics Society, Zuoz, Switzeriand, August 1986, and the 8th IEEE EMBS Conference, Fort Worth, TX, November 1987

H. Wijkstra was with the Department of Electrical Engineering Biomedical Engineering Division, University of Twente, $7500 \mathrm{AE}$ Enschede, The Netherlands. He is now with the Department of Urology, Radboud University Hospital, 6500 HB Nijmegen, The Netherlands.

H. B. K. Boom is with the Department of Electrical Engineering, Biomedical Engineering Division, University of Twente, 7500 AE Enschede, The Netherlands.

IEEE Log Number 8930488

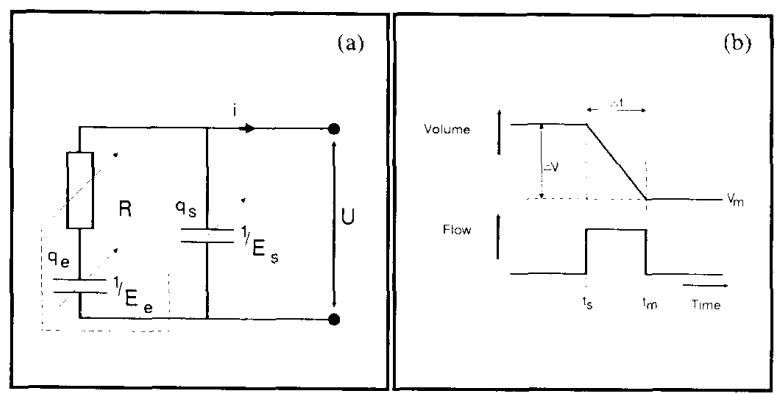

Fig. 1. Panel (a): Electrical analog of the three-component model of left ventricular pressure generation. $E$ : elastance, $1 / E$ : capacitance, $R:$ resistance. $U$ (voltage) represents pressure, $i$ (current) flow, $q_{c}+q$ (charge) volume. The arrows with interconnecting lines represent the common time function $f(t)$. Panel (b): Linear decreasing volume change, constant flow pulse. $t_{s}$ : start of CFP, $t_{m}$ : end of CFP, $\Delta t$ : flow duration, $V_{m}:$ reference volume, $\Delta V:$ stroke volume. During the CFP: $d V / d t=$ -applied flow value

its "volume," despite the presence of series elastance. In addition, resistance follows a time course similar to that of $E(t)$ and $E_{s}(t)$ [38]

At least for nonvarying flow, left ventricular pressurevolume-flow-time relations thus can be summarized by a three-element reference model [Fig. 1 panel (a)]. This model has been shown to describe the pressure response to constant flow pulses [CFP, Fig. 1 panel (b)] before and during the ejection period.

After the end of a constant ejection flow period, in the second isovolumic period, it has been observed that pressure can deviate from the isovolumic pressure expected on the basis of the value of $E(t)$ and for the volume attained at that moment [24], [38], [39]. This effect can partly be explained by the interplay of the series elastance and the resistance (low-pass filter effect). The duration of the effect, however, excludes a complete explanation in this way. For larger flow values, the deviation from the isovolumic pressure will be greater. For this reason, the effect has been characterized as "(flow) deactivation."

Applying a constant ejection flow, the deactivation effect only will be present after the ejection period. However, physiological ejection flow evidently is not constant, and therefore deactivation during the ejection cannot be excluded [39]. Campbell et al. [10] and Lefevre et al. [24] conclude on the basis of simulations the existence of such deactivation. Hunter et al. [15], [16] describe the pressure generation of the ventricle, including a deactivation effect during ejection. Therefore, for an adequate 
representation of the pressure generation by the left ventricle next to elastance, series elastance, and resistance, also a deactivation effect must be incorporated in the model.

This paper presents detailed quantitative information on the effects of deactivation on generated pressure as obtained with the flow-clamp technique. Deactivation will be defined with the help of the three-element reference model, and will be shown to be quantitatively related to applied flow parameters.

\section{THEORY}

We will make use of the reference model of Fig. 1(a) which gives the electrical analog. All the system components vary in time according to the same time function (which is the isovolumic pressure curve). In addition, the resistance and the series elastance are allowed to be volume dependent [30], [32], [38]. The equations describing the model of Fig. 1(a) are

$$
\begin{gathered}
\frac{d V_{e}(t)}{d t}+\frac{E_{e}(t)+E_{s}(t, V)}{R(t, V)} \cdot V_{e}(t)=\frac{E_{s}(t, V)}{R(t, V)} \cdot V(t) \\
P(t, V)=E_{s}(t, V) \cdot\left[V(t)-V_{e}(t)\right]
\end{gathered}
$$

where $V(t)$ is left ventricular volume, $V_{e}(t)$ is volume of the elastance $E_{e}(t), R(t, V)$ is the resistance, and $E_{s}(t$, $V)$ is series elastance. $P(t, V)$ is left ventricular pressure. This model has been verified experimentally during the application of constant flow pulses [38].

The time dependence of the model components is given by $[38]$

$$
\begin{aligned}
E_{e}(t) & =\gamma_{e} \cdot f(t) \\
E_{s}(t, V) & =\gamma_{s}(V) \cdot f(t) \\
R(t, V) & =\rho(V) \cdot f(t) .
\end{aligned}
$$

If (3) is substituted in (1), all time functions in the components cancel. We write (2) as

$$
P(t, V)=\gamma_{s}(V) \cdot f(t) \cdot\left[V(t)-V_{e}(t)\right] .
$$

When volume is constant, $\left(V_{m}\right),(4)$ becomes

$$
P_{\text {iso }}\left(t, V_{m}\right)=\left(\gamma_{s}\left(V_{m}\right) / / \gamma_{e}\right) \cdot f(t) \cdot V_{m}
$$

where $\left(\gamma_{s}\left(V_{m}\right) / / \gamma_{e}\right)=\gamma_{s}\left(V_{m}\right) \cdot \gamma_{e} /\left(\gamma_{s}\left(V_{m}\right)+\gamma_{e}\right)$.

If we neglect the so-called "dead" volume $V_{d}$, (5) represents the time-varying elastance concept [29].

During a CFP, the pressure given by (4) is decreasing due to the decreasing volume and due to the decreasing effect of the applied flow. The last effect includes a transient caused by switching on of the flow which, in turn, is associated with the series elastance $\gamma_{s}(V)$ and which is determined by (1).

Following the end of the CFP ( $\left.t>t_{m}\right)$, another transient effect associated with the switching off of flow is predicted by the model. After ejection, in the second isovolumic period, (1) can be solved simply since volume is constant. This results in

$$
\begin{aligned}
V_{e}(t> & \left.t_{m}, V_{m}\right) \\
= & {\left[V_{e}\left(t_{m}\right)-\frac{\gamma_{s}\left(V_{m}\right)}{\gamma_{s}\left(V_{m}\right)+\gamma_{e}^{\prime}} \cdot V_{m}\right] \cdot e^{-\left(t-t_{m}\right) / \tau_{d}} } \\
& +\frac{\gamma_{s}\left(V_{m}\right)}{\gamma_{s}\left(V_{m}\right)+\gamma_{e}^{\prime}} \cdot V_{m} .
\end{aligned}
$$

In (6), the possibility has been introduced that elastance $\gamma_{e}$ after the CFP has been changed to $\gamma_{e}^{\prime}$. This has been suggested by [24], [25], [39]. $V_{e}\left(t_{m}\right)$ is volume on the elastance $E_{e}$ at $t=t_{m}$. The time constant $\tau_{d}=\rho /\left(\gamma_{e}^{\prime}+\right.$ $\left.\gamma_{s}\right)$.

With (2),

$$
\begin{aligned}
P(t> & \left.t_{m}, V_{m}\right) \\
= & \left(\gamma_{s}\left(V_{m}\right) / / \gamma_{e}^{\prime}\right) \cdot f(t) \cdot V_{m} \\
& -f(t) \cdot \gamma_{s}\left(V_{m}\right) \cdot\left[V_{e}\left(t_{m}\right)-\frac{\gamma_{s}\left(V_{m}\right)}{\gamma_{s}\left(V_{m}\right)+\gamma_{e}^{\prime}} \cdot V_{m}\right] \\
& \cdot e^{-\left(t-t_{m}\right) / \tau_{d}}
\end{aligned}
$$

The first term on the right represents isovolumic pressure at volume $V_{m}$ when no CFP is present. The second represents the transient exponential caused by switching off the flow.

We now define a normalized pressure as $\Pi(t)=P / P_{\text {iso }}$. Thus, dividing (7) by the isovolumic pressure at $V=V_{m}$ (5), the model predicts

$$
\begin{aligned}
\Pi(t)= & \frac{P\left(t>t_{m}, V_{m}\right)}{P_{\mathrm{iso}}\left(t>t_{m}, V_{m}\right)}=\alpha-\left[\frac{\gamma_{s}\left(V_{m}\right)+\gamma_{e}}{\gamma_{e}}\right. \\
& \left.\cdot \frac{V_{e}\left(t_{m}\right)}{V_{m}}-\frac{\gamma_{s}\left(V_{m}\right)}{\gamma_{e}}-(1-\alpha)\right] \cdot e^{-\left(t-t_{m}\right) / \tau_{d}}
\end{aligned}
$$

where

$$
\alpha=\frac{\gamma_{s}\left(V_{m}\right)+\gamma_{e}}{\gamma_{s}\left(V_{m}\right)+\gamma_{e}^{\prime}} \cdot \frac{\gamma_{e}^{\prime}}{\gamma_{e}} .
$$

Thus, the time function $f(t)$ cancels. In Fig. 2, a curve as predicted by (8) is sketched. During the first isovolumic period, $\Pi(t)$ [dividing (4) by (5)] is constant. According to (1) and (2), during the CFP, $\Pi(t)$, normalized pressure, decreases as a result of both volume and flow effects. After the end of the CFP, $\Pi(t)$ is reestablished to a level determined by the value of $\alpha$. Since $\alpha$ was defined by a relative decrease of $\gamma_{e}$ resulting from the existence of a CFP, we will denote $(1-\alpha)$ as (flow) deactivation. Before, during, and after the CFP, the time function $f(t)$ cancels in the $\Pi(t)$ curve.

Since the model on which (8) is based was constructed from CFP experiments, we used experimentally CFP's for validating (8). 


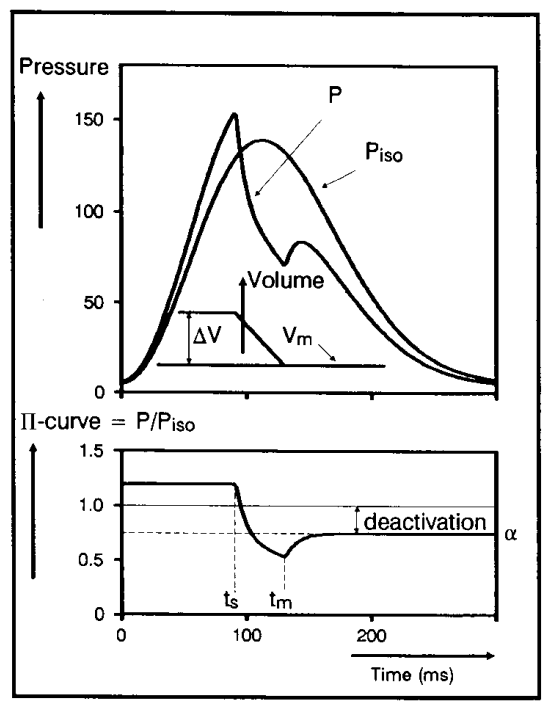

Fig. 2. Upper panel: Sketch of theoretical pressures according to the threeelement model. $P$ : by applying a constant flow pulse, $P_{\text {iso }}$ isovolumic pressure at volume $V_{m}$. Lower panel: Theoretical $\Pi$ curve $=P / P_{\text {iso. }}$. End value $\alpha$ : "activity index," deactivation $(1-\alpha)$.

The switching-on time constant $\tau=\rho /\left(\gamma_{e}+\gamma_{s}\right)$ has been assessed in the literature [6], [38]. The time constant $\tau_{d}$ in (8) in principle depends on deactivation. However, since $\gamma_{s} \gg \gamma_{e}$, (8) predicts the switching-off time constant $\tau_{d}$ to be only slightly greater than $\tau$.

\section{Methods}

\section{Physiological Preparation}

A total of 11 rabbits, weighing $1.9-3.2 \mathrm{~kg}$. were used for the experiments. Most details of the preparation have been fully described elsewhere [38]. The rabbits were anesthetized with a combination of Hypnorm $(10 \mathrm{mg}$ fluanison $/ \mathrm{ml}, 0.2 \mathrm{mg}$ fentanyl base $/ \mathrm{ml} ; 1 \mathrm{ml} / \mathrm{kg}$ initial dose I.M. ) and atropine sulphate $(0.5 \mathrm{mg} / \mathrm{ml} ; 0.4 \mathrm{ml} / \mathrm{kg}$ I.M.). Adequate anesthesia was maintained with additional doses of Hypnorm $(0.1 \mathrm{ml})$ given intravenously when needed. Artificial respiration was maintained during the preparation. After midsternal thoracotomy and administration of heparin ( 5000 I.U. $/ \mathrm{ml} ; 0.5 \mathrm{ml} / \mathrm{kg} \mathrm{I}$.V.) and bleeding, a suitable cannula was inserted in the aorta and ligated close to the aortic valves. After quick complete excision of the heart, the aortic cannula was connected to a perfusion system (see Fig. 3). In two experiments, the aortic valves were damaged by the cannula, resulting in leakage. In these cases, the aorta was completely removed and two thin catheters (diameter $1.0-1.5 \mathrm{~mm}$ ) were inserted into the two coronary arteries and connected to the perfusion system. The aorta was closed with tissue adhesive (Histoacryl blue) at the place of the aortic valves. No difference could be detected in the results with or without the use of catheters. Coronary perfusion during the experiment was maintained at a constant pressure of $75 \mathrm{mmHg}$. In nine experiments, the perfusion fluid was a Tyrode-like solution with the following composition in

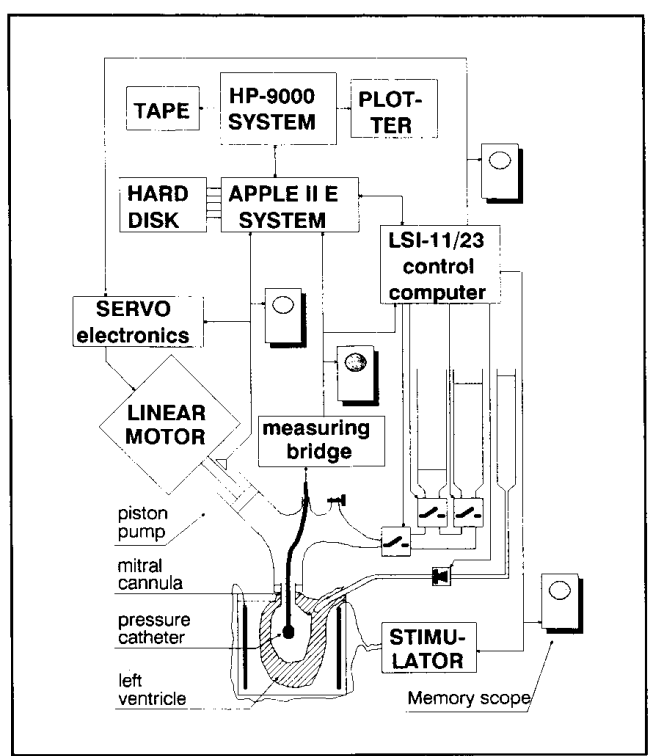

Fig. 3. Experimental setup.

meq/1: $\mathrm{Na}^{+}$156.6, $\mathrm{Ca}^{2+} 2.2, \mathrm{~K}^{+}$5.6, $\mathrm{Cl}^{-}$143.6, $\mathrm{HCO}_{3}^{-} 21.4, \mathrm{H}_{2} \mathrm{PO}_{4}^{-} 1.5$, and glucose 11.7. In two experiments, an FC-43 emulsion (Perfluorochemical Artificial Blood, Green Cross Corporation) [12], [31] was added to the perfusate. The perfusate was oxygenated by gassing with a mixture of 95 percent $\mathrm{O}_{2}$ and 5 percent $\mathrm{CO}_{2}$. Its $\mathrm{pH}$ was $7.37 \pm 0.08\left(\right.$ mean $\pm \mathrm{SD}$ ) at $37^{\circ} \mathrm{C}$. The perfusate was not recirculated. After connection of the aorta cannula to the perfusion system, most of the atria was removed, and a closely fitting cannula was snapped into the mitral orifice. The cannula served to connect the left ventricle to a servo-driven pump system (see below).

At the end of the experiment, the heart (left and right ventricle only) was dried lightly with filter paper and weighed. The averaged heart weight was $6.0 \pm 0.4 \mathrm{~g}$ (mean $\pm \mathrm{SD}$ ).

\section{Experimental Setup}

The experiments were carried out with a computer-controlled setup drawn schematically in Fig. 3. Most of it has been described elsewhere in detail [38]. A piston pump, driven by a linear motor, was connected to the left ventricle by way of a rigid, perfusion fluid-filled, glass assembly and the mitral cannula. Linear piston movements represented constant rated changes of ventricular volume. The linear motor was driven by an electronic servosystem with position and velocity feedback. The range of this volume actuator was $9 \mathrm{ml}$.

Intraventricular pressure was measured with a catheter tip pressure transducer (Honeywell/Philips Type 9815156-1000) introduced via the mitral cannula. The tip was positioned in the center of the ventricle.

The ventricle was paced using silver plate electrodes on both sides of the heart with a frequency of $2 \mathrm{~Hz}$. Temperature was maintained at $37^{\circ} \mathrm{C}$. 


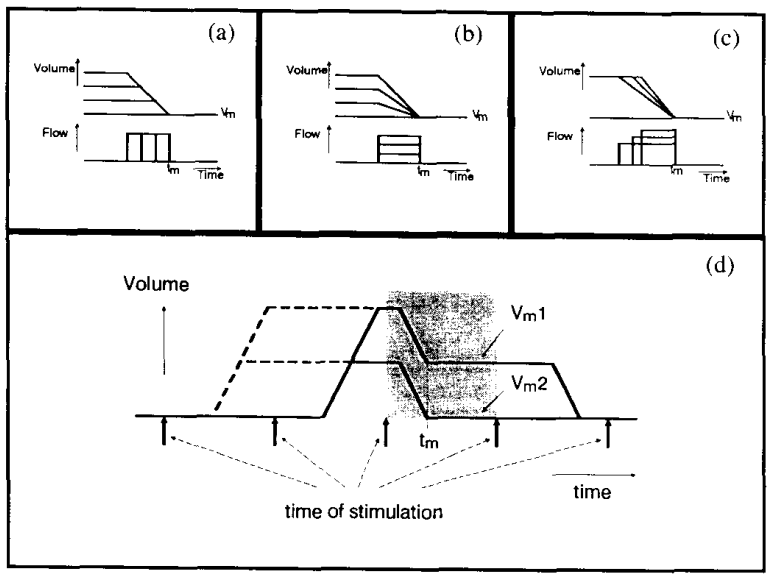

Fig. 4. Panel (a), (b), (c): Constant flow pulses as applied during the experiments with the same end volume $V_{m}$ at the same end time $t_{m}$. (a) Same flow value, different flow duration, different stroke volume. (b) Same flow duration, different flow value, different stroke volume. (c) Constant stroke volume, different flow value, different flow duration. Panel (d): Constant flow pulses at different end volume $V_{m}$ at the same end time $t_{m}$. With interspacing isovolumic beat (broken line) and without (solid line).

Data acquisition was performed by a data acquisition system based on an Apple IIE microcomputer. The pressure and position signals were sampled with a 12 bit $A D$ converter at maximal $1 \mathrm{kHz}$ and stored on a hard disk. After an experiment, the collected data were transferred to a Hewlett-Packard HP-9000 computer for further analysis. The data acquisition system was controlled by the same interactive control language as the experimental setup [3]. Beats were generally sampled with $200 \mathrm{~Hz}$; the beats of special interest were sampled with $1 \mathrm{kHz}$.

\section{Experimental Protocols}

In the experiments, constant flow pulses were applied [Fig. 1, panel (b)], effected by moving the piston with specified, constant speed over a specified interval during systole. In the following, an "experiment" is defined as a series of protocols on one left ventricle where a "protocol" consists of a series of successive CFP's including interspaced stabilizing isovolumic beats.

All experiments started with a stabilization period. After a stable preparation was reached, a number of protocols were performed. Before every CFP in a protocol, (usually) 25 isovolumic beats were used as stabilization beats. The constancy of the maximal pressure obtained during the isovolumic beat preceding the CFP, at volume $V_{m}$, was used as an indicator for stability.

The CFP's used in the experiments are given in Fig. 4(a)-(c). Values of flow, flow duration, and stroke volume could be changed independently of $V_{m}$ and $t_{m}$, which also could be varied. In all protocols, each CFP with equal parameters was applied twice. During the first CFP, volume was increased in the preceding diastole; during the second, an additional isovolumic beat was inserted. In the latter, the beat preceding the CFP was an isovolumic beat at the volume $V_{m}$ plus $\Delta V$. The results of both kinds of CFP's were similar.
Volume $V_{m}$ was changed in two ways. In the first way, end-diastolic pressure was controlled at different values, and thus different end-diastolic volumes were effected. In the second, the volume courses applied were of the form as drawn in Fig. 4(d). In the first beat, only a part of the volume step made in the preceding beat was used. In the second beat, the rest of the volume was withdrawn. No differences in the results could be detected.

During the experiments, the slope of the maximum isovolumic pressure-volume curve, the $E_{\max }$ value, was used as an indicator of contractility.

In the experiments, we did not use an intraventricular balloon for reasons described elsewhere [38]. Thus, we had no simple way to measure the absolute ventricular volume. In this paper, we use the term end volume $V_{m}$ as relative to a reference volume. By controlling end-diastolic pressure or by using volume changes drawn in Fig. $4(d)$, we could change this (relative) end volume $V_{m}$. The constancy of the reference volume at constant end-diastolic pressure was estimated by the constancy of the maximum isovolumic pressure.

\section{RESULTS}

In 11 experiments, we applied a total of 908 CFP's. In the protocols, we systematically changed the values of the flow (range: $0 \cdots 21.2 \mathrm{ml} / \mathrm{s}$ ), flow duration (range: 0 $\cdots 50 \mathrm{~ms})$, stroke volume $(0 \cdots 0.61 \mathrm{ml})$, end time of flow $t_{m}(75 \cdots 150 \mathrm{~ms})$, and end volume $V_{m}(-0.3$ - $0.3 \mathrm{ml}$ relative to the reference volume).

Pressure curves measured with the application of constant flow pulses are given in Figs. 5 and 6. In Fig. 5, all CFP's used had the same flow duration and different flow values, and thus different stroke volumes. Pressure curves with CFP's with constant flow but different duration are given in Fig. 6. The experimental $\Pi_{e}(V, t)$ curves, obtained by dividing the sampled pressure values by the corresponding preceding isovolumic pressure values, are also given in the figures. These experimental measured $\Pi_{e}$ curves behave quantitatively according to the theoretical $\Pi$ curve (Fig. 2, "Theory'). Before the CFP's, the $\Pi_{e}$ curve is constant at a value greater than 1 ; during the CFP's, the $\Pi_{e}$ curve is decreasing. After the CFP, Figs. 5 and 6 show that also in the experiment, the $\Pi_{e}$ value stays constant, and thus forms a plateau in accordance with (8). Usually, the plateau persisted until a moment when the $\Pi_{e}$ curve abruptly deviated from the plateau (Figs. 5, 6).

We tested the prediction by the model that in the experiment also the $\Pi_{e}$ curve after the CFP is exponential and determined by a time constant about the value of the switching-on time constant of the flow. For this, we fitted the calculated experimental $\Pi_{e}$ curves after the CFP with an exponential (see Figs. 5, 6), and calculated the time constant $\tau_{d}$ and the end value $\alpha$. The exponential fitting procedure was performed as follows. Sampled pressure values were divided by the corresponding preceding isovolumic pressure values at $V_{m}$. In the resulting $\Pi_{e}$ curve, the end of the CFP and the start and end time of the plateau were detected automatically by software, and cor- 


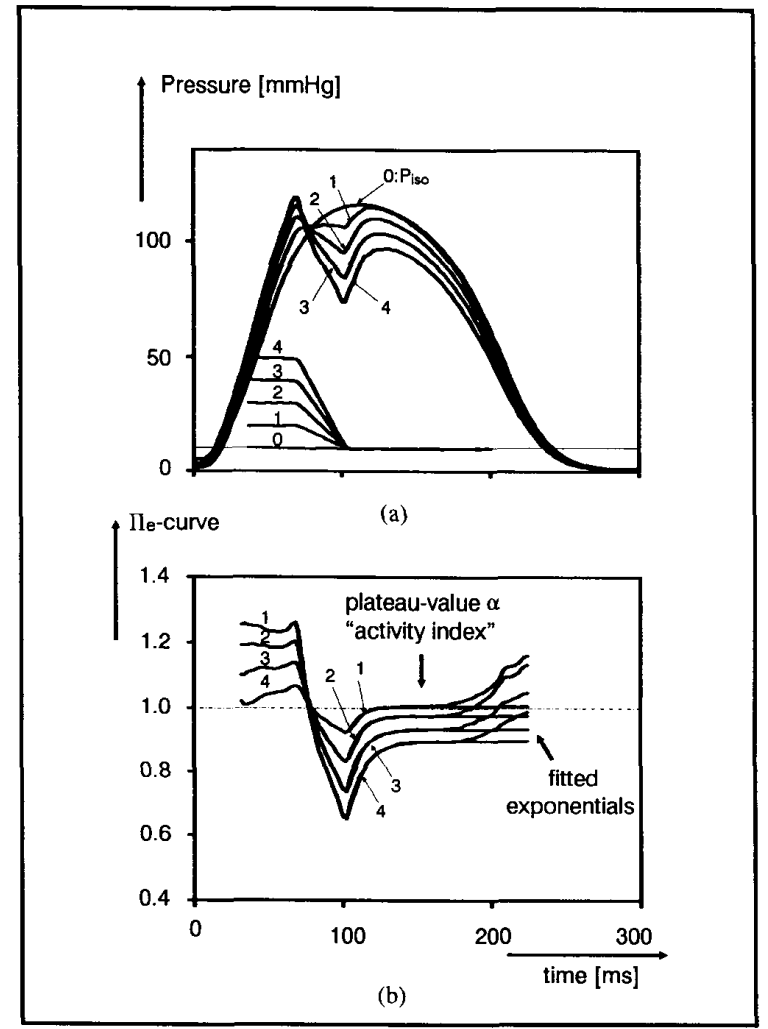

Fig. 5. Panel (a): Typical result of experimental measured pressure curves by applying constant flow pulses with different flow values and constant flow duration. Flow duration is $33 \mathrm{~ms}$. Flow values: $0,3,6, \cdots, 12$ $\mathrm{ml} / \mathrm{s}$. Ejected volume: $0,0.099,0.198, \cdots, 0.396 \mathrm{ml}$. End time of CFP's is $105 \mathrm{~ms}$. Panel (b): The result of dividing the CFP affected pressure curves by the isovolumic pressure at $V_{m}\left(\Pi_{e}\right.$ curve $)$. Fitted exponentials also are given.

rected if found necessary by visual inspection. The plateau value $(\alpha)$, the mean value between start and end time of the plateau, was used as the asymptotic value of the exponential, while the value of $\Pi_{e}$ at $t=t_{m}$ was used as a start value. Linear regression was performed on the logarithm of the $\Pi_{e}$ values. Since the start and end values were known, only the time constant $t_{d}$ had to be calculated from the slope of the regression line.

For the end time of the plateau, we found a value of $195 \pm 19 \mathrm{~ms}$ (mean \pm SD). We also calculated the time of the maximal negative peak of the first time derivative of the isovolumic pressure, and found $196 \pm 12 \mathrm{~ms}$ (mean \pm SD). Thus, the plateau in the $\Pi_{e}$ curve exists until after the normal time of end ejection [1]. The mean plateau length of all calculated $\Pi_{e}$ curves was $53 \mathrm{~ms}$; the maximal length was $140 \mathrm{~ms}$. We calculated the root mean-squared differences between the fitted exponential and calculated $\Pi_{e}$ curve, and found a value of $0.0071 \pm 0.0028$ (mean $\pm \mathrm{SD}$ ). In the calculation, all sampled points between the end of the CFP until the end of the plateau were used. For the part until the start of the plateau, we found $0.0062 \pm$ 0.0039 ; for the plateau, we found $0.0074 \pm 0.0029$. We concluded that the parameters $\alpha$ and $\tau_{d}$ in (8) are experimentally well-definable values.

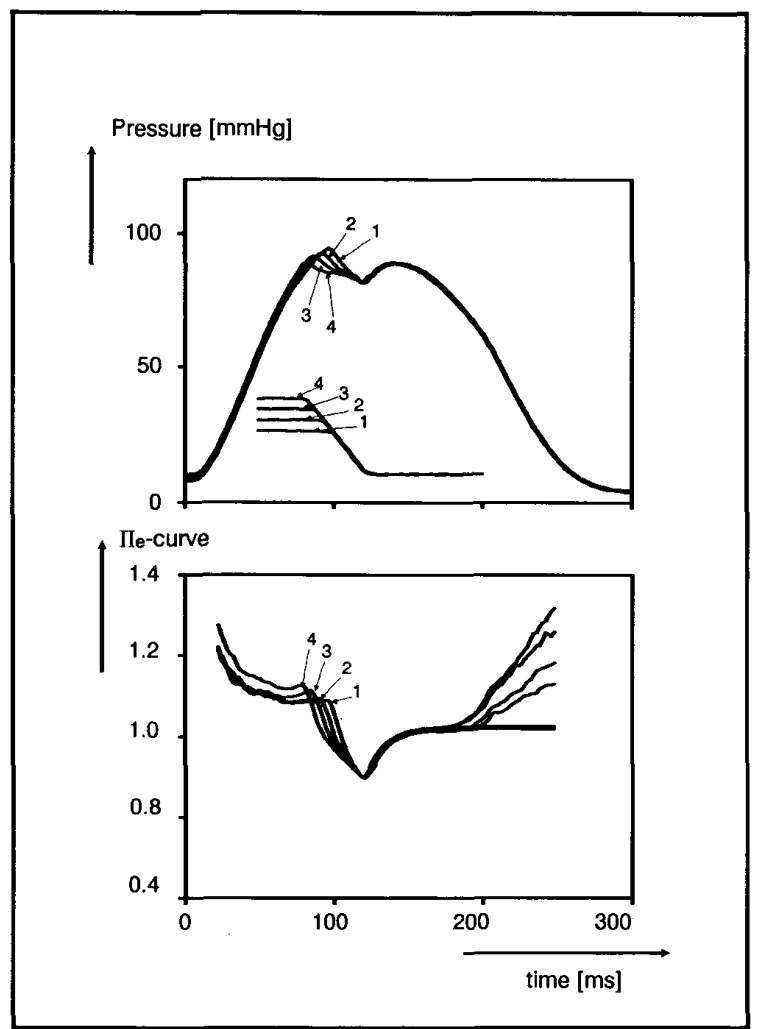

Fig. 6. CFP's with same flow value and different flow duration. Flow is 6 $\mathrm{ml} / \mathrm{s}$. Flow duration: 24, 30, 36, $43 \mathrm{~ms}$. End time of CFP's is $120 \mathrm{~ms}$.

\section{Deactivation Plateau Value}

Generally, we found deactivation $(1-\alpha)$ to be dependent on the value of $\Pi_{e}$ at time $t_{m}\left(\Pi_{\dot{e}}\left(t_{m}\right)\right)$. If at the end of ejection a lower $\Pi_{e}\left(t_{m}\right)$ was reached, as a result of, e.g., a higher flow value, a smaller value of $\alpha$, and thus a greater deactivation effect was found.

An $\alpha, \Pi_{e}\left(t_{m}\right)$ relation for one protocol is given in Fig. 7. Below a certain value of $\Pi_{e}\left(t_{m}\right)$, here 0.75 , a linear relation between $\alpha$ and $\Pi_{e}\left(t_{m}\right)$ is reached. For values of $\Pi_{e}\left(t_{m}\right)$ near one, the value of $\alpha$ is greater than 1 ; thus, deactivation is negative, which according to our terminology, could be denoted by "superactivation." Apparently, the ventricle is capable of generating pressure in excess of what would be generated at the same volume without a preceding volume decrease (see "Discussion"').

From all $\Pi_{e}$ curves, for the part where $\alpha$ and $\Pi_{e}\left(t_{m}\right)$ "by eye" were linearly related, the linear regression lines were calculated, and were used to characterize the behavior of $\alpha$.

We found $\alpha$ to depend uniquely on $\mathrm{II}_{e}\left(t_{m}\right)$. The value of $\Pi_{e}\left(t_{m}\right)$ in turn depends on both flow and flow duration, with the additional property that when flow duration is greater than $30 \mathrm{~ms}, \Pi_{e}\left(t_{m}\right)$ only depends on the flow value [38]. Thus, for the shorter flow duration, the same $\Pi_{e}\left(t_{m}\right)$ value can be reached by different flow and flow-duration values. In Fig. $8, \alpha$ is plotted against $\Pi_{e}\left(t_{m}\right)$, using dif- 


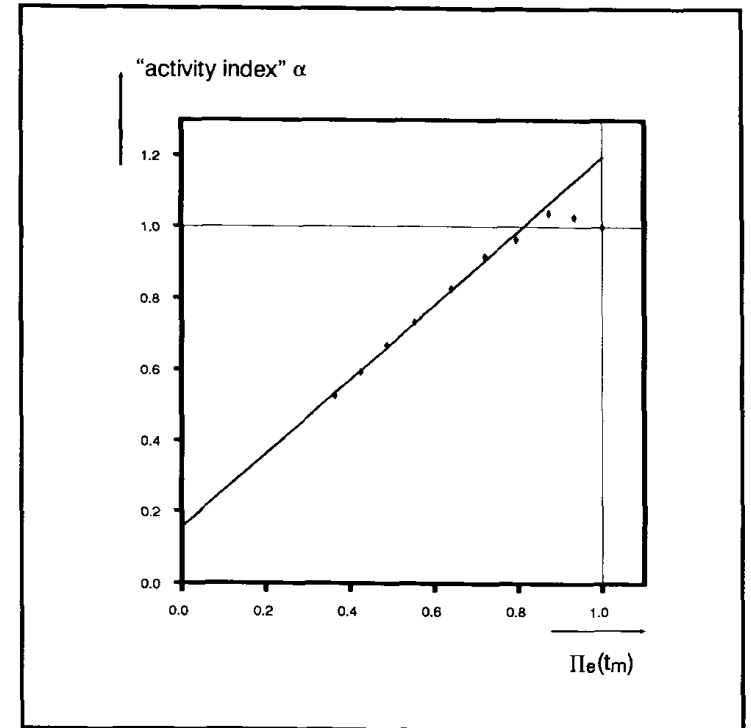

Fig. 7. Example of $\alpha$, "activity index," related to the value of the $\Pi$. curve at $t=t_{m}$. Also, the linear regression line (for $\Pi_{r^{\prime}}\left(t_{m}\right)$ values less than 0.75$)$ is given. Protocol with different flow values $(0,2,4,6, \cdots$, $18 \mathrm{ml} / \mathrm{s})$ and constant flow duration $(30 \mathrm{~ms})$. For the regression line was found: $\alpha=1.04 \cdot \Pi_{e}\left(t_{m}\right)+0.16$. For $\Pi_{e}\left(t_{m}\right)$ values less than 0.75 for the correlation coefficient, a value of 0.998 was found.

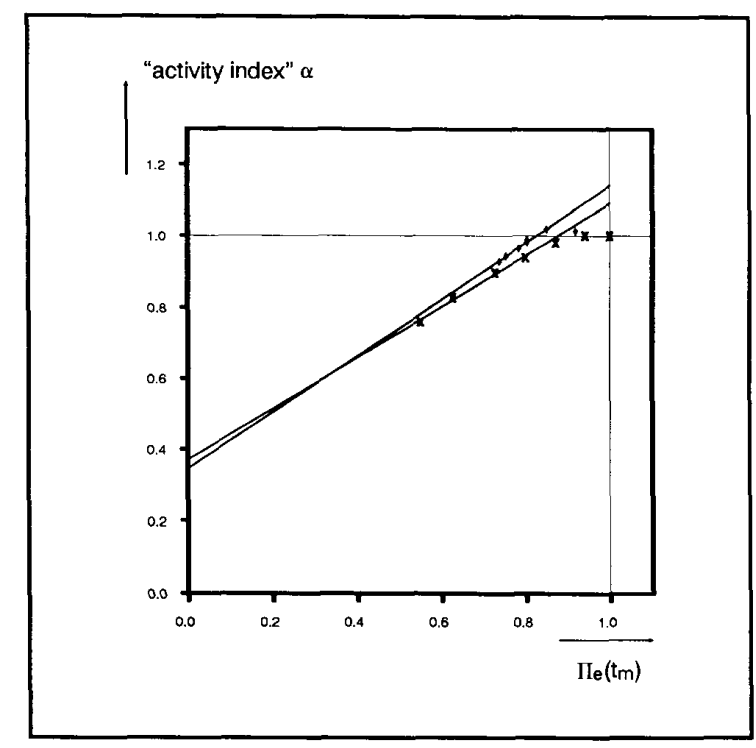

Fig. 8. $\alpha$ related to $\Pi_{e}\left(t_{m}\right)$ using two different protocols. One protocol with same flow value $(\bullet: 12 \mathrm{ml} / \mathrm{s})$ and different flow duration $(0,6$, $12, \cdots, 42 \mathrm{~ms})$, the other $(\times)$ with CFP's with different flow values $(0,3,6, \cdots, 18 \mathrm{ml} / \mathrm{s})$ and constant flow duration $(30 \mathrm{~ms})$. For the linear regression lines was found: $\alpha=0.79 \cdot \Pi_{e}\left(t_{m}\right)+0.35(\bullet)$ and $\alpha=0.72 \cdot \Pi_{e}\left(t_{m}\right)+0.37(\times)$. Correlation coefficients for the linear part: 0.996 and 0.996 .

ferent protocols with different flow and flow duration, with $t_{m}$ and $V_{m}$ constant. Also, the regression lines and the correlation coefficients are given. The figure shows that in this case, the $\alpha, \Pi\left(t_{m}\right)$ relation is independent of the individual flow and flow-duration values.

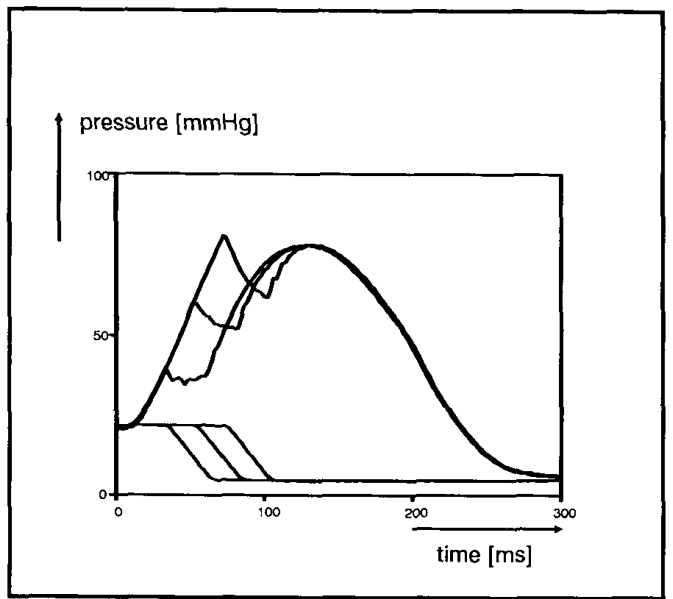

Fig. 9. Experimental measured pressure curves by applying the same constant flow pulse at different $t_{m}(100,120,140 \mathrm{~ms})$. Used CFP: flow value $=12 \mathrm{ml} / \mathrm{s}$, flow duration $=36 \mathrm{~ms}$. After the CFP, following a transient effect, all pressure curves reach a common path, indicating the same degree of deactivation

According to the theory and the experimental finding that all the components vary in time according to one time function $f(t)$, the $\Pi_{e}\left(t_{m}\right)$ values are predicted to be independent of the end time $t_{m}$. It is therefore also predicted that $\alpha$ is independent of $t_{m}$. In two experiments, we examined the relation between $\alpha$ and $\Pi_{e}\left(t_{m}\right)$ at different end time $t_{m}$. Fig. 9 shows an example of experimental measured pressure curves by applying the same CFP's which end at different $t_{m}$ in systole. Following the CFP's, after a transient effect as described above, all pressure curves indeed reach a common path, indicating the same degree of deactivation.

Previous findings [32], [38] found a linear volume dependence of the resistance. Then, according to the theory, $\Pi_{e}\left(t_{m}\right)$ is independent of the end volume $V_{m}$. In three experiments, we measured $\alpha$ related to $\Pi_{e}\left(t_{m}\right)$ at different end volumes $V_{m}$. Fig. 10 shows an example of the relation between $\alpha$ and $\Pi_{e}\left(t_{m}\right)$ at different volumes $V_{m}$. Clearly, this $\alpha, \Pi\left(t_{m}\right)$ relation is not affected by changing $V_{m}$.

Averaging all the results from all 11 experiments, we found for the regression coefficient $0.85 \pm 0.16$, for the extrapolated $\Pi_{e}\left(t_{m}\right)=1$ intercept $1.14 \pm 0.08$, and for extrapolated $\Pi_{e}\left(t_{m}\right)=0$ intercept $0.29 \pm 0.11$, all values in mean $\pm \mathrm{SD}$. A value of $0.973 \pm 0.031$ ( mean $\pm \mathrm{SD}$ ) was found for the correlation coefficient.

From the small SD values, we conclude that the relation between $\alpha$ and $\Pi_{e}\left(t_{m}\right)$ does not depend on flow, flow duration, stroke volume, end time $t_{m}$, and end volume $V_{m}$. This is despite the fact that $\Pi_{e}\left(t_{m}\right)$ and thus, indirectly, $\alpha$ do depend on flow, flow duration, and thus stroke volume.

\section{Deactivation Time Constant}

Fig. 11 shows a typical result of the relation between the time constant $\tau_{d}$ and flow duration. In total, we used 25 protocols in eight experiments to examine the relationship. The flow values range was 6 to $12.4 \mathrm{ml} / \mathrm{s}$, flow 


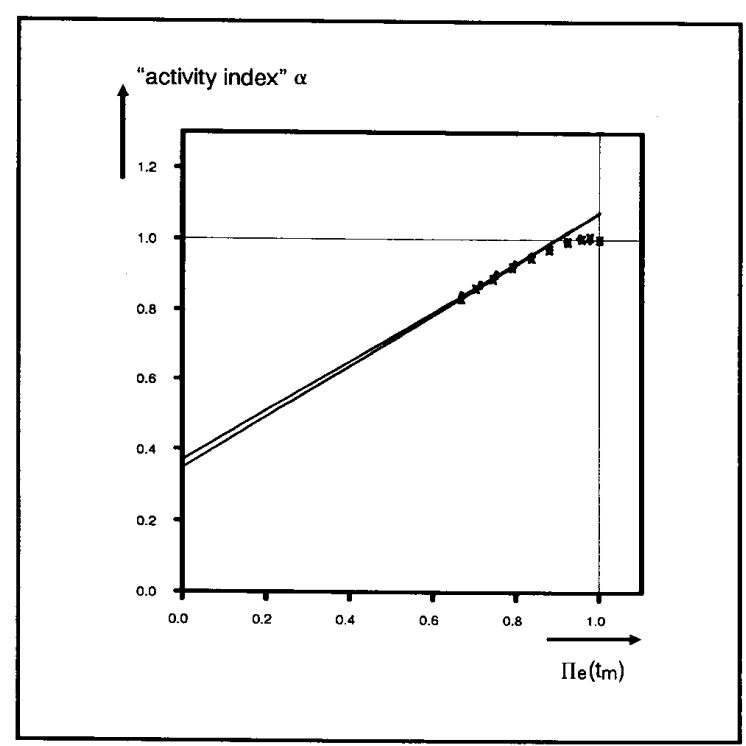

Fig. 10. $\alpha$ related to $\Pi_{e}\left(t_{m}\right)$ at different end volume $V_{m}(0,0.254 \mathrm{ml})$ relative to the reference volume, using the same protocols: flow duration $=30 \mathrm{~ms}$, flow $=0,2,4, \cdots, 18 \mathrm{ml} / \mathrm{s}$. For the linear regression lines was found: $\alpha=0.71 \cdot \Pi_{e}\left(t_{m}\right)+0.37$ and $\alpha=0.73 \cdot \Pi_{e}\left(t_{m}\right)+0.35$

Correlation coefficients for the linear part: 0.999 and 0.998 .

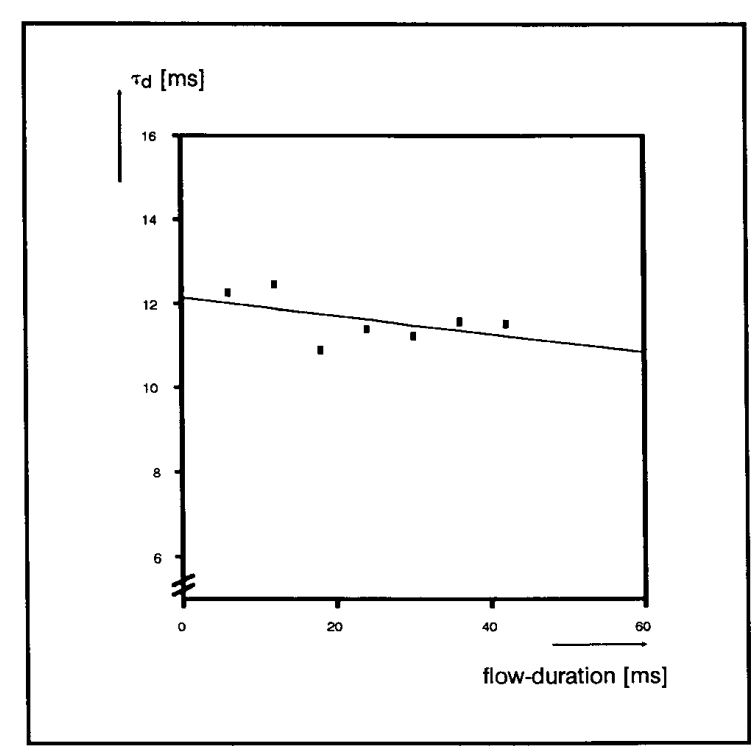

Fig. 11. $\tau_{d}$ related to flow duration. Flow $=12 \mathrm{ml} / \mathrm{s}$, flow duration $=0$ $6,12, \cdots, 42$. For the linear regression line was found: $\tau_{d}=-0.022$ $\Delta t+12.14$

duration ranged from 0 to $60 \mathrm{~ms}$, and end time $t_{m}$ between 81 and $150 \mathrm{~ms}$. In all 25 protocols, we calculated the linear regression, and found a value of $-0.038 \pm 0.042$ (mean $\pm \mathrm{SD}$ ) for the regression coefficient, which is statistically different from zero (two-sided $t$ test, hypothesis mean $=0, P=0.001$ ).

Using CFP's with a constant flow duration but different flow values, we examined the relation between $\tau_{d}$ and applied flow value in 26 protocols in nine experiments. Fig. 12 gives a typical result. Flow values were 1 to 21.18

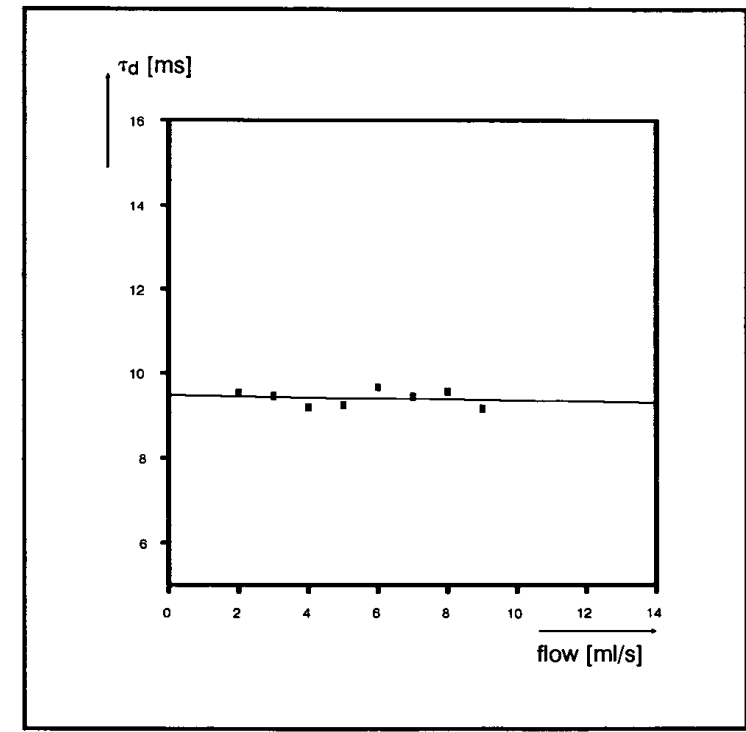

Fig. 12. $\tau_{d}$ related to flow value. Flow duration $=33 \mathrm{~ms}$, flow $=2,3$, $\cdots, 9 \mathrm{ml} / \mathrm{s}$. For the regression line was found: $\tau_{d}=-0.012 \cdot \Phi$ +9.46 .

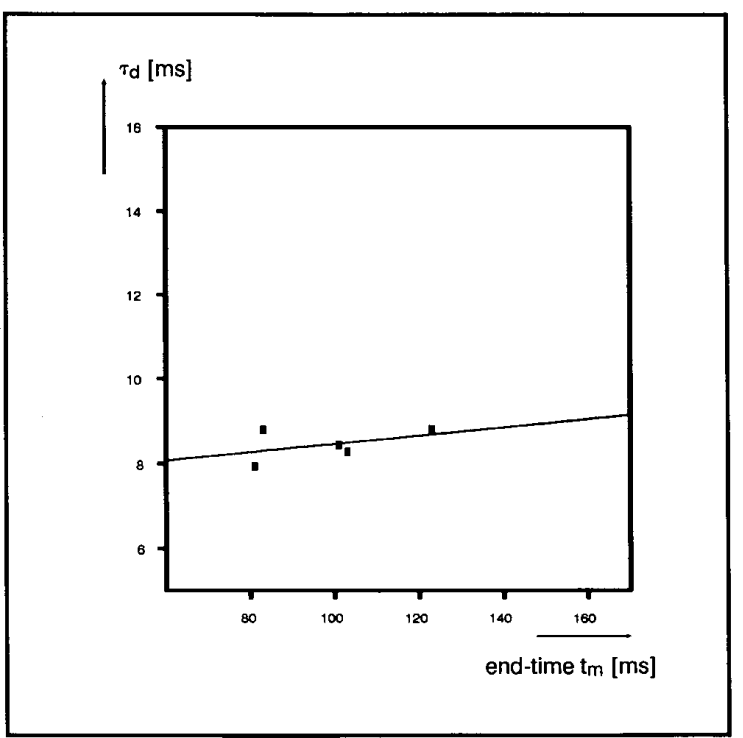

Fig. 13. $\tau_{d}$ related to $t_{m}$. For the regression line was found: $\tau_{d}=-0.0098$

$$
\cdot t_{m}+7.49
$$

$\mathrm{ml} / \mathrm{s}$, flow duration usually $33 \mathrm{~ms}$, and end time $t_{m}$ ranged from 81 to $150 \mathrm{~ms}$. Linear regression was calculated, and for the regression coefficient, a value of $-0.09 \pm 0.25$ (mean $\pm S D$ ) was found, which was not significantly different from zero (two-sided $t$ test, $P>0.08$ ).

In two experiments, we performed the same protocols at different end time $t_{m}$ in systole. In total, 23 protocols were used to examine this relation; one example is given in Fig. 13. Again, linear regression was calculated, and we found for the regression coefficient $0.003 \pm 0.03$ (mean $\pm \mathrm{SD})$, not significantly different from zero $(P>$ $0.6)$. 
End volume $V_{m}$ was changed in three experiments. In total, 26 protocols were used. Linear regression was performed, and we found a value of $1.7 \pm 5.3$ for the regression coefficient, not significantly different from zero $(P$ $>0.1$ ).

We conclude that the time constant $\tau_{d}$ is statistically independent of flow, stroke volume, end time $t_{m}$, and end volume $V_{m}$. Only the regression coefficient of the relation between $\tau_{d}$ and flow duration is statistically different from zero, but has a small value ( $30 \mathrm{~ms}$ results in a change in $\tau_{d}$ of $1.1 \mathrm{~ms}$ ).

For the total averaged value of $\tau_{d}, 10.44 \pm 0.09 \mathrm{~ms}$ (mean \pm SEM) was found. In all the experiments, the nontime-varying switching-on time constant $\tau$ of the threeelement model [6] was also calculated in the way described in [6]. This resulted in a value of $10.35 \pm 0.26$ ms (mean \pm SEM). Thus, we could not detect a significant difference between the two time constants (two-sided $t$ test, $P>0.3$ ).

\section{DISCUSSION}

In the literature, a depressive effect on the pressure during and after normal ejection has been shown to be present [10], [15], [16], [25], [32], [38], although no commonly used definition of this deactivation phenomenon can be found. Hunter et al. [15], [16] define "deactivation" by assuming that the corresponding pressure decrease is identical for both withdrawal and infusion of a volume step. Earlier studies [6]-[9], [32], [38] support the validity of the reference model of Fig. 1(a), both before and during constant flow pulses. After the end of the CFP, pressure deviates from this model prediction. According to the model, (8) with $\alpha=1$, after a CFP pressure, would return to the isovolumic pressure. Our definition of deactivation $(1-\alpha)$ is based on the difference between isovolumic pressure and pressure after a CFP. The present study shows that problems arising in earlier work in relating deactivation to other contraction parameters in the cardiac cycle arose since ejection flow had not been held constant, and pressure had not been normalized by isovolumic pressure. By using constant flow pulses, we found deactivation to be uniquely related to the pressure at the end of the CFP, and thus also to the flow value. Ensuing from our definition, and the fact that the three-element reference model is valid during constant flow pulses, deactivation stays zero after the flow starts, and appears when flow stops. Experiments with two cascaded CFP's [39] show that deactivation only exists during the ejection phase when flow has been decreasing. Simulations by Campbell et al. [10] support this finding also for normal ejection. These authors found the greatest deviations from model simulations in late ejection, thus when flow is decreasing.

The present study shows "deactivation"' as represented by the quantity $(1-\alpha)$ to be constant during a great part of the systole. Until the moment of the maximal negative first derivative of the isovolumic pressure, the parameter $\alpha$ is constant, while earliest CFP's we used had an end time $t_{m}$ value of $75 \mathrm{~ms}$. Thus, "deactivation" is a constant phenomenon during the normal ejection period [1].

With CFP's, elastic, resistive, and deactivation effects can be measured separately. Comparison of the results from CFP experiments to experiments in which other volume interventions were used (varying flow) is difficult because then all these effects are simultaneously present.

Normal ejection can be thought to be assembled by many short CFP's in series. Doing so, a summation of deactivation terms during the time the flows are decreasing, and thus in late ejection, can be expected [39]. The summation effect, however, is unknown, and its result may be greater than the deactivation values found in this paper by using one CFP. This remains to be further investigated.

Deactivation as experimentally observed in the current study is in accordance with (7) and (8). These expressions, for $\alpha \neq 1$, in turn can be interpretated as a change of the end-ejection pressure-volume relation (see the end of "Discussion"). Much attention has been given to the end-systolic pressure-volume relation [4], [19], [22], [26], [27], [29], [33]-[37] and the results have been contradicting. When resistive and/or deactivation effects are present, these in itself have a decreasing effect on the pressure. Thus, when limiting the description of systolic pressure to a varying elastance, any resistive and/or deactivation effect will be incorporated into the elastic effect. This results, for instance, in an $E_{\max }$ value dependent on the shape of ejection (Shroff et al. [32, Fig. 6]). Comparably, when using a description including a resistance, any deactivation effect present will influence both the elastance and resistance [25]. This could be the reason why such models have no great predictive value in describing normal ejection [10].

Suga et al. [33], [35], [36] used exponentially shaped volume changes, arranged in the way we applied constant flow pulses, and they measured the end-systolic pressurevolume relation. After ejection, pressure stayed below the corresponding isovolumic pressure [33, Fig. 4], indicating deactivation. Suga et al. correlated pressure decrease and end-systolic flow value, peak flow value, and $\int-d V$, and found a correlation between pressure decrease and end-systolic pressure the highest. However, as a result of the continually decreasing flow, it can be surmised from our study that a deactivation effect during the ejection also will have occurred. Correspondingly, therefore, this high correlation can be expected from our experiments.

Shroff et al. [32] used constant flow pulses with constant flow duration in the blood perfused canine left ventricle, but did not make an explicit effort to quantify the deactivation phenomenon. Their Figs. 1-4, however, show flow or $\Pi_{e}\left(t_{m}\right)$ dependent deactivation. Simulating normal ejection patterns with a model consisting of elastance and resistance values measured with the CFP technique, these authors demonstrated excessive simulated pressure values at end systole, the excess being linearly related to the measured pressure at end systole. We also found, above a certain value, a linear relation between 
deactivation and the pressure at the end of the CFP. However, since the effect of the series elastance and effects of the nonconstant flow are not incorporated in the simulation model of Shroff et al., quantitative comparison is difficult.

Hunter et al. [15], [16] used rounded flow pulses to quantify elastance, resistance, and deactivation effects and also defined a "volume influence factor." They used small flow pulses as a modification of the impulse-response method. They stated that less rapid flow pulses have a smaller deactivation effect, which we also found. The deactivation effect these authors found also in the first part of the flow pulse where flow is increasing differs from our findings as a result of a different definition of deactivation.

The time independency of $\alpha$ during a major part of systole can also be observed in the results of Kaam [21, pp. $76,163]$. It seems, however, at variance with the results of Hunter et al. [16] who found a deactivation effect with a maximal value after time of peak isovolumic pressure. An essential condition for the time invariance of deactivation found in our study is that deactivated pressure and nondeactivated isovolumic pressure after the end of ejection (CFP) at the same volumes are compared. In contrast, Hunter et al. compared pressure at preejection volume to pressure after ejection. It is, however, well known [5] that at greater volumes, peak isovolumic pressure as well as the descending limb of the isovolumic pressure curve are shifted to later times. In our model, this corresponds to a change of $f(t)$. Hunter et al. used moderately large volume steps (comparable to $\approx 0.15 \mathrm{ml}$ in the rabbit left ventricle), and thus the use of preejecting isovolumic pressure will result in an appreciably greater pressure difference, and hence a greater deactivation effect for times after peak isovolumic pressure. In fact, we eliminated the influence of $f(t)$ on volume by dividing pressure by isovolumic pressure at end-ejection volume. Therefore, our finding that $\alpha$ is independent of time in systole $\left(t_{m}\right)$ is not contradictory.

The finding of elastic, resistive, and deactivation effects reflects three major parameters of ventricular dynamics. It may be questioned whether such parameters can also be identified in cardiac muscle mechanics. Elastance would reflect the force-length relation, and resistance the force-velocity relation. Likewise, deactivation would correspond to a reduced capability of force development, resulting from previous shortening velocity. On the muscular level, it has been shown that length changes cause a depression of the ability to generate force, which does not contradict a velocity-determined deactivation effect since length changes imply shortening velocity. It seems therefore likely that deactivation is a property of cardiac muscle.

It is accepted that the time course of intracellular calcium concentration determines the time course of a mechanical systole [20]. Thus, the common time function $f(t)$ in the model may be closely linked to the overall intracellular calcium concentration time course. It has been suggested that shortening (ejection) decreases the binding of calcium to troponin [2], [14], thus releasing calcium from the sarcomeres. An increased uptake of calcium by the SR could be the result. Therefore, after ejection, the amount of calcium present in the sarcoplasm may be lower, and force cannot be regenerated to the isometric level, resulting in deactivation. This mechanism would imply deactivation to be built up during the flow pulse. Clearly, this would not affect the observation that resistance, with or without deactivation having partly incorporated, behaves in the way as described by (1).

Force generated by a sarcomere is believed to be the result of the formation of cross bridges between the actin and myosin filaments [18]. The simplest way to describe this is by approximating it by a first-order process [5]. Then the breaking and formation of bridges are determined by one single time constant. The present finding of one same time constant for switching on and switching off of flow supports this assumption.

Deactivation has been observed in cardiac muscle. Edman et al. [13] used a quick release method on rabbit papillary muscle, applied with and without damping of the lever movements. With no damping, deactivation was the greatest, and the authors conclude that the more changes in shortening velocity arise, the greater the resulting deactivation is. Also, the contractile process appeared to be not depressed by accelerations. Rather, the depressant effect had, probably, to be attributed to the decelerations. These findings correspond closely to our finding that deactivation on ventricular level is related to the flow (or $\left.\Pi_{e}\left(t_{m}\right)\right)$ and only exists after flow has been decreasing [39].

ter Keurs et al. [23] examined deactivation in rat cardiac muscle. They imposed triangle-shaped sarcomere length changes; thus, after the change, the sarcomere length had the same value as before the change. From their Fig. 5(c), it is observed that after a length change, developed force is decreased. The shape of the deactivated force is comparable to the force curve without length change, corresponding to our results on the ventricular level.

From the present study, it can be concluded that (8) describes the "deactivation phenomenon" satisfactorily. According to (8) and (8a), $\alpha<1$ is equivalent to the switching on of an additional capacitor in the three-element model of Fig. 1(a), thus giving rise to the model of Fig. 14. The value of $\gamma_{e}$ is changed to $\gamma_{e}^{\prime}=\left(\gamma_{e} \cdot \gamma_{d}\right) /\left(\gamma_{e}\right.$ $\left.+\gamma_{d}\right)$. This was already suggested by Lefevre et al. [24], [25]. The time constant $\tau_{d}$ is related to $\tau$ by

$$
\frac{\tau_{d}}{\tau}=\frac{\gamma_{e}+\gamma_{s}}{\gamma_{e}^{\prime}+\gamma_{s}}
$$

Since it has been found [38] that $\gamma_{s} \gg \gamma_{e}$, it follows that $\tau_{d}$ is only slightly greater than $\tau$. This is in accordance with experimental values for $\tau(=10.35 \pm 0.26 \mathrm{~ms})$ and $\tau_{d}(=10.44 \pm 0.09 \mathrm{~ms})$, although the difference between $\tau$ and $\tau_{d}$ is not significant. 


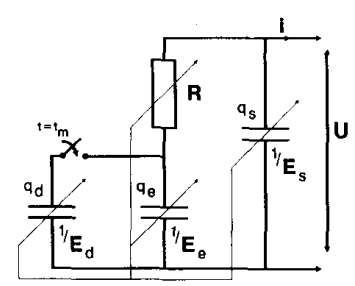

Fig. 14. Reference model for left ventricular pressure generation, including "deactivation" (see "Discussion"), Symbols: See Fig. 1. Total charge (volume) after CFP: $q_{e}+q_{s}+q_{d}$.

In the present study, we found that after small flow pulses, pressure can exceed isovolumic pressure at the same volume. This "superactivation" also is present in the results of Hunter et al. [15], [16] and Campbell et al. [10]. Nwasokwa et al. [28] found this phenomenon in the in situ dog papillary muscle. Thus, ejection can have a positive effect on pressure [11], [17]. It has been discussed that deactivation and superactivation represent different mechanisms in the muscle [17]. The existence of superactivation was not studied in detail in this paper. The model of Fig. 14 can describe this effect by introducing before $t_{m}$ a charge at the deactivation capacitor $1 / E_{d}$.

\section{ACKNOWLEDGMENT}

The authors would like to thank Prof. Dr. K. Sagawa for the free provision of the FC-43 emulsion and Prof. Dr. N. Westerhof for his helpful criticism in the preparation of this paper.

\section{REFERENCES}

[1] F. L. Abel, "Maximal negative $d P / d t$ as an indicator of end of systole," Amer. J. Physiol., vol. 240, pp. H676-H679, 1981.

[2] D. J. Allen and S. Kurihara, "The effects of intracellular calcium transients in mammalian cardiac muscle," J. Physiol. London, vol. 327, pp. 79-94, 1982.

[3] J. A. van Alste, A. L. Schoute, S. R. Vaartjes, and H. B. K. Boom, "Interactive control of isolated heart experiments,", in Computers in Cardiology, Seattle, WA, IEEE Computer Society, 1983, pp. 255258.

[4] J. Baan and E. T. van der Velde, "Sensitivity of left ventricular endsystolic pressure-volume relation to type of loading intervention in dogs," Circ. Res., vol. 62, pp. 1247-1258, 1987.

[5] H. B. K. Boom, J. J. D. van der Gon, J. H. M. Nieuwenhuijs, and P. Schiereck, "Cardiac contractility: Actin-myosin interaction as measured from left ventricular pressure curve," European J. Car diol., vol. 1, no. 2, pp. 217-224, 1973

[6] H. B. K. Boom, S. R. Vaartjes, and H. Wijkstra, "Time varying elastance, resistance and series elastance of the left ventricle resulting in an non-varying time constant,"' in Proc. 6th Int. Conf. Workshop CSDS, Philadelphia, PA, 1984, pp. 304-307.

[7] H. B. K. Boom and H. Wijkstra, "A three element model of the left ventricle and the quantification of flow deactivation," in Proc. XIV ICMBE and VII ICMP, vol. 23, suppl. part 2, Espoo, Finland, 1985, pp. 1309-1311.

[8] - "Ventricular elastance and resistance after decreasing flow," in Proc. 8th Conf. EMBS/IEEE, Fort Worth, TX, 1986.

[9] - , "Ventricular mechanics: Facts and models," in Proc. 9th Annu. Conf. BME-IEEE, Boston, MA, 1987.

[10] K. B. Campbell, J. A. Ringo, G. G. Knowlen, R. D. Kirkpatrick, and S. L. Schmidt, "Validation of optional elastance-resistance left ventricle pump models," Amer. J. Physiol., vol. 251, pp. H382H397, 1986
[11] K. B. Campbell, R. D. Kirkpatrick, and J. A. Ringo, "Failing of elastance-resistance left ventricular pump model," in Proc. 9th Annu. Conf. BME-IEEE, Boston, MA, 1987.

[12] J. M. Chemnitius, W. Burger, and J. Bing, "Crystalloid and perfluorochemical perfusates in an isolated working rabbit heart preparation," Amer. J. Physiol., vol. 249, pp. H285-H292, 1985.

[13] K. A. P. Edman and E. Nilsson, "Relationships between force and velocity of shortening in rabbit papillary muscle," Acta Physiol. Scand., vol. 85, pp. 488-500, 1985 .

[14] A. M. Gordon and E. B. Ridgway, "Calcium transients and relaxation in single muscle fibers," European J. Cardiol., vol. 7, suppl., pp. $27-34,1978$

[15] W. C. Hunter, J. S. Janicki, K. T. Weber, and A. Noordergraaf, "Flow pulse response: A new method for the characterization of ventricular mechanics,"'Amer. J. Physiol., vol. 237, pp, H282-H292, 1979.

[16] - "Systolic mechanical properties of the left ventricle: Effects of volume and contractile state," Circ. Res., vol. 52, pp. 319-327, 1983.

[17] W. C. Hunter, "Ventricular contraction modelled as a balance between positive and negative influences on ejection," in Proc. 9th Annu. Conf. BME-IEEE, Boston, MA, 1987.

[18] A. F. Huxley, "Muscle structure and theories of contraction," Prog. Biophys. Chem., vol. 7, pp. 255-318, 1957.

[19] Y. Igarashi and H. Suga, "Assessment of slope of end-systolic pressure-volume line of in situ dog heart," Amer. J. Physiol., vol. 250 pp. H685-H692, 1986.

[20] B. R. Jewell, "A reexamination of the influence of muscle length on myocardial performance," Circ. Res., vol. 40, pp. 221-230, 1977.

[21] F. van Kaam, "Towards a sarcomere basis for heart muscle contraction," Ph.D. dissertation, Utrecht, The Netherlands, 1986.

[22] S. Kaseda, H. Tomoike, I. Ogata, and M. Nakamura, "End-systolic pressure-volume, pressure-length, and stress-strain relations in canine hearts,"' Amer. J. Physiol, , vol. 249, pp. H648-H654, 1985.

[23] H. E. D. J. ter Keurs, W. H. Rijnsburger, and R. van Heuningen, "Restoring forces and relaxation of cat cardiac muscle," European Heart J., vol. 1, suppl. A, pp. 67-80, 1980

[24] J. Lefevre, J. Melbin, H. Pouleur, A. Charlier, and A. Noordergraaf, "Ventricular flow-dependent deactivation: Is it an artefact, a detrimental effect or a useful mechanism?," in Proc. 6th Int. Conf. Workshop CSDS, Philadelphia, PA, 1984, pp. 17-20.

[25] J. Lefevre, J. Melbin, A. Tavenier, F. Messemaeckers, Y. Rijkmans, H. Pouleur, A. Charlier, and A. Noordergraaf, "Left ventricular resistance and deactivation," presented at the 38th ACEMB, 1985.

[26] W. L. Maughan, K. Sunagawa, D. Burkhoff, and K. Sagawa, "Effect of arterial impedance changes on the end-systolic pressure-volume relation," Circ. Res., vol. 54, pp. 595-602, 1984.

[27] W. L. Maughan, K. Sunagawa, and K. Sagawa, "Effect of load, contractility heart rate and ischemia on the left ventricular pressure-volume relationship," in Proc. 6th Int. Conf. Workshop CSDS, Philadelphia, PA, 1984, pp. 178-181.

[28] O. Nwasokwa, K. Sagawa, and H. Suga, "Short-term memory in the in situ canine myocardium," Amer. J. Physiol., vol. 247, pp. H8H16, 1984.

[29] K. Sagawa, H. Suga, A. A. Shoukas, and K. M. Bakalar, "Endsystolic pressure/volume ratio: A new index of ventricular contractility,"'Amer. J. Cardiol., vol. 40, pp. 748-753, 1977.

[30] P. Schiereck and H. B. K. Boom, "Left ventricular stiffness: Dependency on time and inotropic state," Pflügers Arch., vol. 374, pp. 135-143, 1978.

[31] L. D. Segel and S. V. Rendig, "Isolated working rat heart perfusion with perfluorochemical emulsion Fluosol-43," Amer. J. Physiol., vol. 242, pp. H485-H489, 1982.

[32] S. G. Shroff, J. S. Janicki, and K. T. Weber, "Evidence and quantitation of left ventricular systolic resistance, ' Amer. J. Physiol., vol 249 , pp. H358-H370, 1985.

[33] H. Suga, A. Kitabatake, and K. Sagawa, "End-systolic pressure determines stroke volume from fixed end-diastolic volume in the isolated canine left ventricle under a constant contractile state," Circ. Res., vol. 44, pp. 238-249, 1979.

[34] H. Suga and K. Sagawa, "Instantaneous pressure-volume relationships and their ratio in the excised, supported canine left ventricle,' Circ. Res., vol. 35, pp. 117-126, 1974.

[35] H. Suga, K. Sagawa, and L. Demer, "Determinants of instantaneous pressure in canine left ventricle," Circ. Res., vol. 47, pp. 256, 1980.

[36] H. Suga and K. Yamakoshi, "Effects of stroke volume and velocity of ejection on end-systolic pressure of canine left ventricle," Circ. Res., vol. 40, pp. 445-450, 1977 
[37] R. R. Taylor, J. W. Covell, and J. Ross, Jr., "Volume-tension diagrams of ejecting and isovolumic contractions in the left ventricle,' Amer. J. Physiol, vol. 216, pp. 1097-1102, 1969

[38] S. R. Vaartjes and H. B. K. Boom, "Left ventricular internal resistance and unloaded ejection flow assessed from pressure-flow relations: A flow-clamp study on isolated rabbit hearts," Circ. Res., vol. 60 , pp. 727-737, 1987

[39] H. Wijkstra and H. B. K. Boom, "Deactivation and a five element model of the left ventricle," in Proc. 8th Conf. Workshop CSCD, Zuoz, Switzerland, 1986

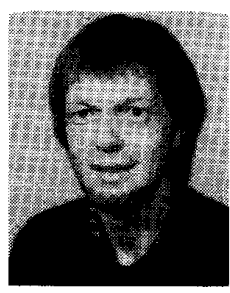

Hessel Wijkstra was born in Noordwijk, The Netherlands, in 1955. After polytechnics, he studied electrical engineering at the University of Twente, The Netherlands, and received the M.Sc. degree in 1984. His M.Sc. thesis was on the relation between constant unloaded ejection flow and contractility in the rabbit left ventricle. In 1984 he began research at the Biomedical Engineering $\mathrm{Di}$ vision, University of Twente. This regarded deactivation in the rabbit left ventricle for which he received the Ph.D. degree in 1989.

He is now with the Department of Urology, Radbou University Hospital, Nijmegen, The Netherlands.

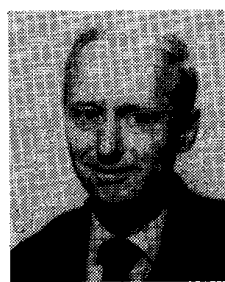

Herman B. K. Boom was trained as a Medical Physicist at the University of Utrecht, The Netherlands, where he received the Ph.D. degree in 1971.

He joined the Department of Medical Physics and Medical Physiology where he was engaged in research in the field of cardiac mechanics and taught physiology and biophysics. Since 1976 he has held the Chair of Medical Electronics in the Department of Electrical Engineering and is Chairman of the Biomedical Engineering Division, University of Twente, The Netherlands. His research interests are cardiovascular system dynamics, bioelectricity, and rehabilitation technology. 